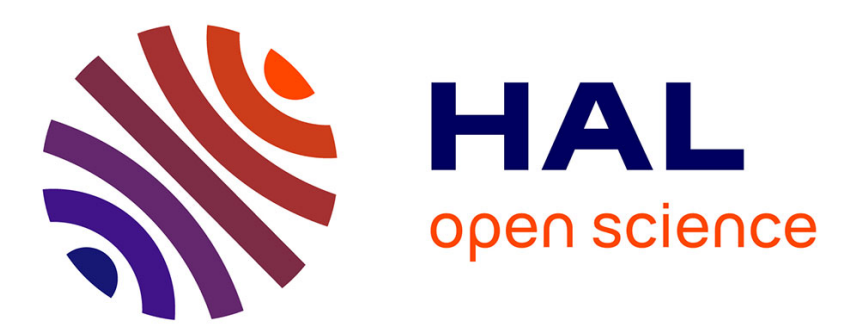

\title{
Méthode générale de calcul thermique d'un système en mouvement avec changement d'état \\ Ph. Cassagne
}

\section{To cite this version:}

Ph. Cassagne. Méthode générale de calcul thermique d'un système en mouvement avec changement d'état. Revue de Physique Appliquée, 1982, 17 (11), pp.737-743. 10.1051/rphysap:019820017011073700 . jpa-00245051

HAL Id: jpa-00245051

https://hal.science/jpa-00245051

Submitted on 1 Jan 1982

HAL is a multi-disciplinary open access archive for the deposit and dissemination of scientific research documents, whether they are published or not. The documents may come from teaching and research institutions in France or abroad, or from public or private research centers.
L'archive ouverte pluridisciplinaire HAL, est destinée au dépôt et à la diffusion de documents scientifiques de niveau recherche, publiés ou non, émanant des établissements d'enseignement et de recherche français ou étrangers, des laboratoires publics ou privés. 


\title{
Méthode générale de calcul thermique d'un système en mouvement avec changement d'état
}

\author{
Ph. Cassagne \\ Equipe Technologie de la Croissance Cristalline, Centre National de la Recherche Scientifique, \\ 1, place Aristide Briand, 92190 Meudon, France
}

(Reçu le 31 mars 1982, révisé le 13 juillet 1982, accepté le 21 juillet 1982)

\begin{abstract}
Résumé. - Dans le cadre du développement d'une méthode de croissance de ruban de silicium, une étude théorique de la forme d'équilibre d'une zone fondue sur la génératrice d'un cylindre en rotation a été entreprise. Pour analyser ce système thermique complexe, une méthode générale de calcul d'un corps en mouvement avec changement d'état a été élaborée. On montrera les applications de cette méthode à un problème unidimensionnel, puis au cas du cylindre en rotation.
\end{abstract}

\begin{abstract}
In the development of a silicium ribbon growth method, the equilibrium shape of a melted zone on the generative line of a rotating cylinder has been investigated. In order to analyse this complex thermal system, a general method to calculate a moving body with change of state has been elaborated. The application of this method to a one-dimensional problem and to a rotating cylinder will be seen.
\end{abstract}

1. Introduction. - L'étude théorique présentée dans cet article a été entreprise dans le cadre du développement d'une nouvelle méthode de croissance de rubans de silicium [1]. La mise au point de cette méthode nécessitait des études théoriques approfondies en thermique et en croissance cristalline, de façon à mieux comprendre la complexité des interactions de tous les paramètres du système. En particulier, la croissance s'effectuant à partir d'une zone fondue formée sur un cylindre de silicium en rotation, il était nécessaire de comprendre le comportement thermique d'une telle zone fondue.

Il s'agit donc de l'étude thermique d'un corps en mouvement avec changement d'état. Un tel système est fort complexe et rares sont les cas où existe une solution analytique [2]. Des méthodes numériques ont été développées sous des hypothèses d'unidimensionnalité, ce qui simplifie beaucoup le problème [3-5]. Zook et Schuldt ont élaboré des modèles partiellement analytiques de ruban à une ou deux dimensions [6]. Il a paru intéressant de mettre au point une méthode numérique générale permettant de traiter tout problème de ce type à une ou deux dimensions. Cette méthode est décrite ci-après, sous sa forme générale et sous une forme simplifiée.

On expose d'abord un modèle préliminaire sans changement d'état, qui simule le comportement d'un cylindre de silicium chauffé sur une génératrice en dessous du point de fusion. Puis on teste la méthode générale dans le cas d'un système unidimensionnel pour lequel existe une solution analytique. Enfin, cette méthode sert à résoudre le problème du cylindre.

2. Effets thermiques de la rotation d'un cylindre chauffé sur une génératrice sans changement d'état. 2.1 Hypothèses. - Le cylindre étudié est supposé parfaitement isolé thermiquement sur ses faces latérales, ce qui permet de ne traiter qu'un problème à deux dimensions. Son rayon $R$ est égal à $7,5 \mathrm{~mm}$. Le flux de chaleur incident est supposé uniformément réparti sur une génératrice de largeur $\delta=3 \mathrm{~mm}$. La longueur $l$ de cette génératrice est arbitraire du fait de la bidimensionnalité : pour fixer les idées et l'ordre de grandeur des puissances mises en jeu, on la supposera égale à $30 \mathrm{~mm}$.

Expérimentalement, le mode de chauffage utilisé est le bombardement électronique. Pour simplifier les calculs, on traitera le silicium comme un corps opaque au rayonnement thermique et au bombardement électronique. En fait, on sait que le silicium est partiellement transparent dans l'infrarouge, et que l'absorption d'un faisceau d'électrons d'énergie de 
l'ordre de $10 \mathrm{keV}$ se produit sur une couche de quelques dizaines à une centaine de microns.

2.2 Mise en ÉQuATIONS. - L'équation générale de conductibilité $: \operatorname{div}(\lambda \operatorname{grad} u)=\rho c \frac{\mathrm{d} u}{\mathrm{~d} t}$ prend la forme suivante dans un repère de coordonnées polaires $(r, \theta)$ en régime permanent :

$$
\lambda \Delta u+\operatorname{grad} \lambda \cdot \operatorname{grad} u=\rho c \omega \frac{\partial u}{\partial \theta}
$$

où $\omega$ est la vitesse de rotation du cylindre et $\lambda=\lambda_{0} / u$ la conductivité thermique du silicium, inversement proportionnelle à sa température absolue $u$.

Soit :

$$
\begin{aligned}
\frac{\lambda_{0}}{u}\left(\frac{\partial^{2} u}{\partial r^{2}}+\frac{1}{r}\right. & \left.\frac{\partial u}{\partial r}+\frac{1}{r^{2}} \frac{\partial^{2} u}{\partial \theta^{2}}\right)- \\
& -\frac{\lambda_{0}}{u^{2}}\left[\left(\frac{\partial u}{\partial r}\right)^{2}+\frac{1}{r^{2}}\left(\frac{\partial u}{\partial \theta}\right)^{2}\right]=\rho c \omega \frac{\partial u}{\partial \theta}
\end{aligned}
$$

Sur toute la circonférence du cylindre, la température doit vérifier la condition aux limites :

$$
-\lambda \frac{\partial u}{\partial r}=\varepsilon \sigma\left(u^{4}-u_{0}^{4}\right)-\Phi_{\mathrm{s}}
$$

$u_{0}:$ température ambiante

$\varepsilon$ : émissivité moyenne

$\Phi_{\mathrm{s}}$ : puissance incidente par unité de surface (nulle dans les régions non bombardées).

2.3 Méthode De RÉSolution. - Du fait de la réunion des caractéristiques suivantes : conductivité fonction de la température, terme de rotation, conditions aux limites non linéaires et discontinues, il paraît exclu de trouver une solution analytique à ce problème. On a eu recours à une méthode de différences finies à maillages variables résolues par une technique de relaxation. Pour une famille de mailtages donnés, on considère que le système a convergé quand le résidu sur une relaxation devient inférieur à une valeur prédéterminée. Pour décider de la convergence absolue d'un calcul, on utilise le critère de la conservation de la puissance.

On peut remarquer que, du fait du maillage de la surface, il existe une incertitude systématique sur la puissance reçue, cette incertitude diminuant avec les dimensions du maillage.

2.4 Résultats. - Les résultats de la simulation sont indiqués sur les figures 1 à 6 . On a choisi une puissance de $180 \mathrm{~W}$ qui correspond au point de fusion $(1683 \mathrm{~K})$ au centre de la région bombardée pour un cylindre immobile de dimensions indiquées précédemment. Les isothermes sont tracés tous les $50 \mathrm{~K}$.

A faible vitesse de rotation $(<1 \mathrm{t} / \mathrm{min}$.) la différence avec le cylindre au repos est peu marquée,

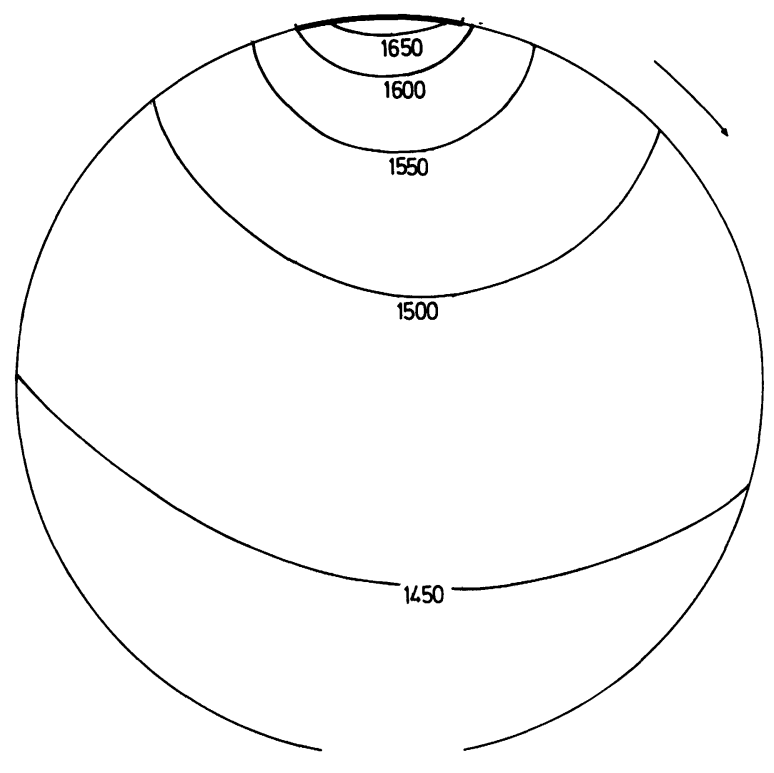

Fig. 1. - Forme des isothermes pour $\Phi=180 \mathrm{~W}$ et $\omega=1 \mathrm{t} / \mathrm{min}$.

[Isotherms shape for $\Phi=180 \mathrm{~W}$ and $\omega=1 \mathrm{t} / \mathrm{min}$.]

surtout dans sa partie la plus chaude ( $\mathrm{t} / \mathrm{min}$., tour par minute).

Jusqu'à $4 \mathrm{t} / \mathrm{min}$. la rotation se traduit par une élévation de température à l'avant de la partie bombardée, et une diminution à l'arrière et au centre. Le gradient orthoradial augmente au contraire à l'arrière de la région bombardée et diminue à l'avant. Les gradients radiaux diminuent très faiblement.

A vitesse plus élevée (jusqu'à $15 \mathrm{t} / \mathrm{min}$., la déformation des isothermes est très prononcée, les tempéra-

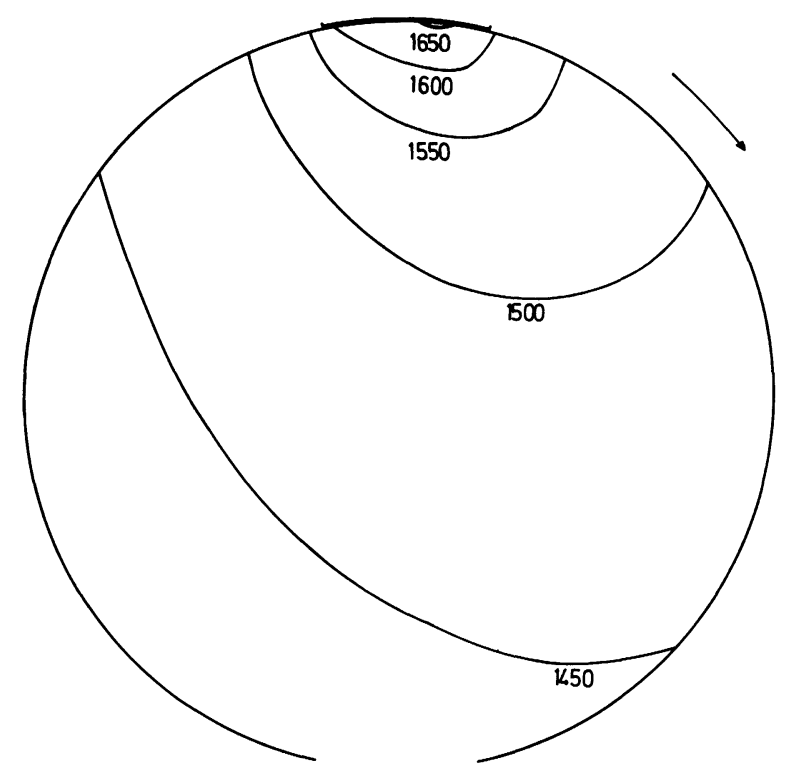

Fig. 2. - Forme des isothermes pour $\Phi=180 \mathrm{~W}$ et $\omega=4 \mathrm{t} / \mathrm{min}$.

[Isotherms shape for $\Phi=180 \mathrm{~W}$ and $\omega=4 \mathrm{t} / \mathrm{min}$.] 


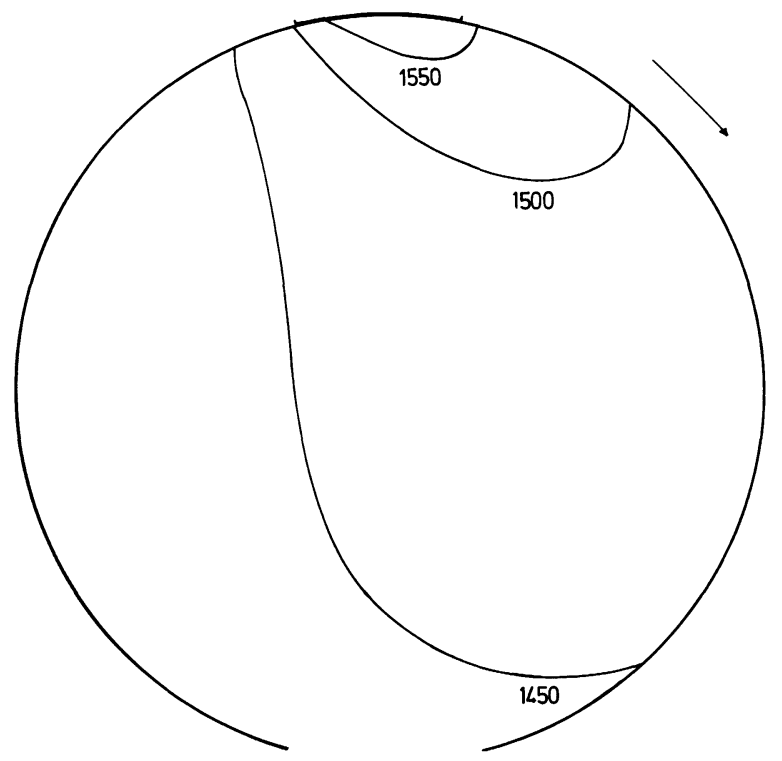

Fig. 3. - Forme des isothermes pour $\Phi=180 \mathrm{~W}$ et $\omega=10 \mathrm{t} / \mathrm{min}$.

[Isotherms shape for $\Phi=180 \mathrm{~W}$ and $\omega=10 \mathrm{t} / \mathrm{min}$.]

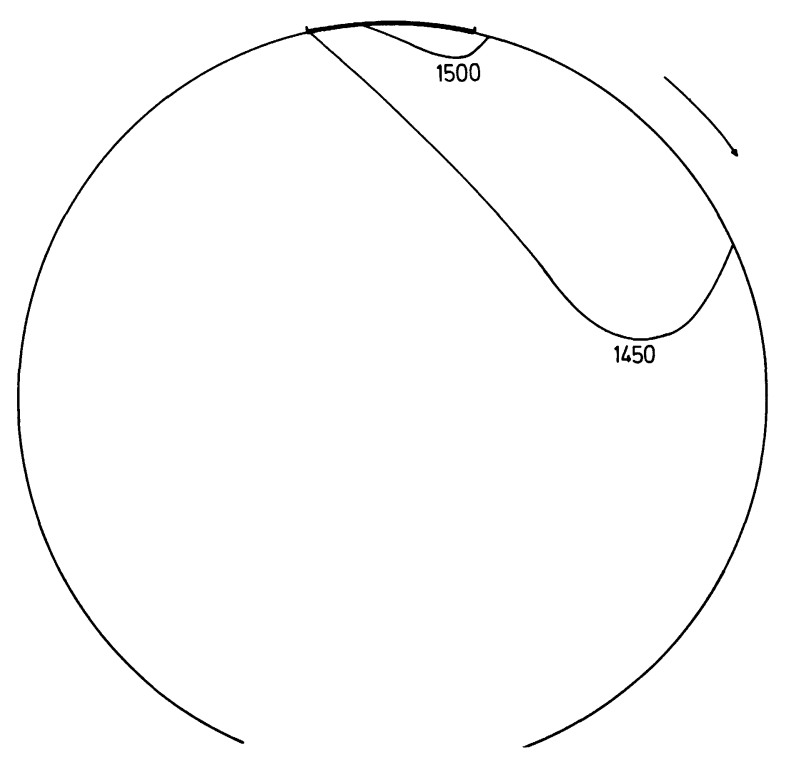

Fig. 5. - Forme des isothermes pour $\Phi=180 \mathrm{~W}$ et $\omega=20 \mathrm{t} / \mathrm{min}$.

[Isotherms shape for $\Phi=180 \mathrm{~W}$ and $\omega=20 \mathrm{t} / \mathrm{min}$.]

tures et les gradients diminuent du fait de l'effet de mélange induit par la rotation.

Enfin, à vitesse très élevée (20-25 t/min.), on observe un changement de concavité des isothermes qui tendent à devenir concentriques, alors que l'effet d'homogénéisation se fait sentir de plus en plus. On peut déterminer facilement par le calcul la vitesse de rotation nécessaire pour que l'écart de température maximum sur le cylindre soit inférieur à $10 \mathrm{~K}, 50 \mathrm{~K}$, etc...

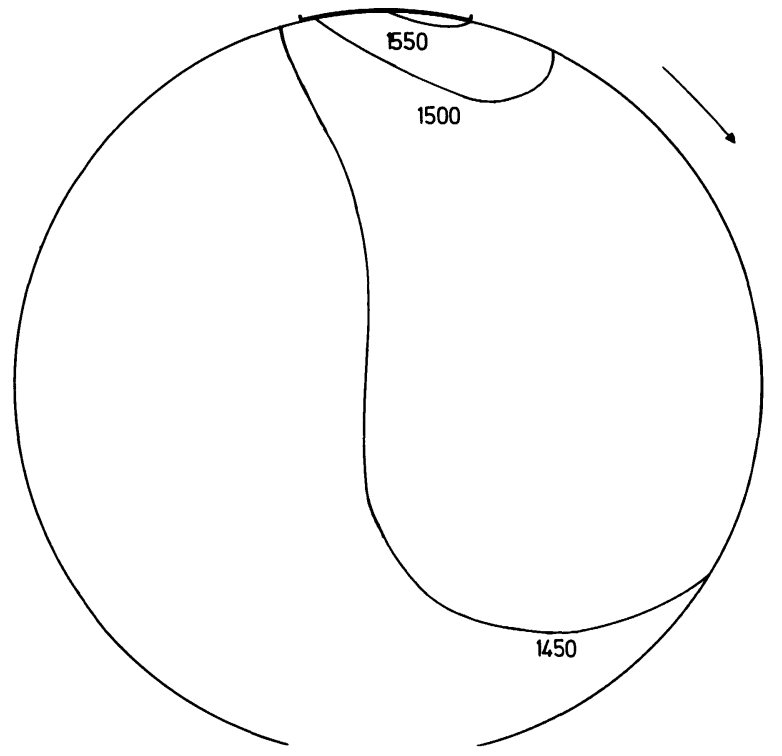

Fig. 4. - Forme des isothermes pour $\Phi=180 \mathrm{~W}$ et $\omega=15 \mathrm{t} / \mathrm{min}$.

[Isotherms shape for $\Phi=180 \mathrm{~W}$ and $\omega=15 \mathrm{t} / \mathrm{min}$.]



Fig. 6. - Forme des isothermes pour $\Phi=180 \mathrm{~W}$ et $\omega=25 \mathrm{t} / \mathrm{min}$.

[Isotherms shape for $\Phi=180 \mathrm{~W}$ and $\omega=25 \mathrm{t} / \mathrm{min}$.]

3. Principes de la méthode générale proposée. 3.1 Position DU PROBLÈME. - On supposera que le liquide a le même champ de vitesses que le solide ce qui revient à négliger au sein du liquide toutes les convections autres que les convections forcées induites par le mouvement du solide. Sous cette hypothèse, les équations d'un système à deux états sont :

— dans le solide : 


$$
\operatorname{div}\left(\lambda_{\mathrm{s}} \operatorname{grad} u\right)=\rho_{\mathrm{s}} c_{\mathrm{s}}\left(\frac{\partial u}{\partial t}+\mathbf{V}_{\mathrm{M}} \cdot \operatorname{grad} u\right)
$$

- dans le liquide :

$$
\operatorname{div}\left(\lambda_{1} \operatorname{grad} u\right)=\rho_{1} c_{1}\left(\frac{\partial u}{\partial t}+\mathbf{V}_{M} \cdot \operatorname{grad} u\right)
$$

- à l'interface :

$$
\left\{\begin{array}{l}
u=u_{\mathrm{F}} \\
-\lambda_{\mathrm{s}}\left(\frac{\partial u}{\partial n}\right)_{\mathrm{s}}=-\lambda_{\mathrm{l}}\left(\frac{\partial u}{\partial n}\right)_{\mathrm{l}}+\rho_{\mathrm{s}} V_{\mathrm{c}}
\end{array}\right.
$$

Les indices s se rapportent au solide, 1 au liquide.

$\mathbf{V}_{\mathbf{M}}$ : vitesse du point $\mathbf{M}$ considéré, par rapport à un repère fixe.

$u_{\mathrm{F}}:$ température de fusion

$L$ : chaleur latente de fusion

$n$ : vecteur unitaire normal à l'interface au point $M$

$V_{\mathrm{c}}$ : vitesse de changement d'état en $\mathrm{M}$, par rapport à un repère fixe.

$V_{\mathrm{c}}=\mathbf{V}_{\mathrm{M}} \cdot \mathbf{n}-v, v=$ vitesse de déplacement de l'interface en $\mathbf{M}$ selon sa normale.

Dans cet article, on se limite volontairement au cas du régime permanent, de sorte que $: \partial u / \partial t=0$ et $V_{\mathrm{c}}=\mathbf{V}_{\mathrm{M}} \cdot \mathbf{n}$.

$V_{\mathrm{c}}$ étant du signe de $\left(\frac{\partial u}{\partial n}\right)_{\mathrm{s}}$ s'il y a fusion, de $-\left(\frac{\partial u}{\partial n}\right)_{\mathrm{s}}$ s'il y a cristallisation, la relation (3) est valable pour un interface de fusion comme pour un interface de cristallisation.

3.2 MÉTHODE GÉNÉRALE. - La méthode consiste à résoudre simultanément les équations (1), (2), (3) mises sous forme de différences finies par une technique de relaxations élaborée de la manière suivante (Fig. 7) :

- on part d'une distribution de températures quelconque (toutefois une distribution judicieusement choisie peut accélérer la convergence);

- l'itération $n$ étant achevée, on cherche par un double balayage en $x$ et en $y$ les points d'intersection



Fig. 7. - Méthode générale : principe de calcul.

[General method : the calculation principle.] du maillage avec le ou les interfaces (on peut créer de nouveaux points ou conserver les points du maillage initial si celui-ci est suffisamment fin). La zone à calculer est alors divisée en solide, liquide et interfaces. En chaque point des interfaces on a besoin de connaître l'angle $\alpha$, défini figure 7 , pour calculer $\partial u / \partial n$ et V.n. L'angle $\alpha$ est déterminé par $\operatorname{tg} \alpha=\frac{\partial u / \partial y}{\partial u / \partial x}$. On en déduit :

$$
\frac{\partial u}{\partial n}=\frac{\partial u}{\partial x} \cos \alpha+\frac{\partial u}{\partial y} \sin \alpha
$$

et $\mathbf{V} . \mathbf{n}=V \cos \alpha$. On peut alors calculer la distribution de températures à l'itération $n+1$ par les relations (1), (2), (3);

- on arrête le calcul quand le résidu sur une relaxation devient inférieur à une valeur prédéterminée. On dira qu'un calcul est convergent si l'énergie est conservée avec une précision fixée initialement.

L'erreur de méthode inhérente à ce procédé de calcul vient du fait qu'il conduit à " épaissir » l'interface, cet épaississement tendant vers zéro avec les dimensions du maillage.

La difficulté du calcul vient de ce qu'on ne connaît a priori ni la position, ni la forme des interfaces qui dépendent à la fois des sources de chaleur et des vitesses. Toutefois, dans certains problèmes pratiques, on peut, connaissant la position des interfaces, prévoir approximativement leur forme. Il est alors possible de donner une version simplifiée de la méthode précédente.

3.3 MÉThOde SIMPLIFIÉE. - Supposons que la position de l'interface ne dépende que de deux paramètres : $V$ (vitesse du solide) et $\Phi$ (flux de chaleur incident sur le liquide) (voir Fig. 8).

$\mathrm{Si}$ l'on fixe arbitrairement l'interface dans une position $P_{0}$, on peut résoudre l'équation (1) dans le solide, en déduire $(\partial u / \partial n)_{s}$, puis par (3) $(\partial u / \partial n)_{1}=f(V)$. On peut alors résoudre (2) en ajustant $\Phi$ de manière à obtenir une valeur préétablie de $(\partial u / \partial n)_{1}$ pour la même position de l'interface.

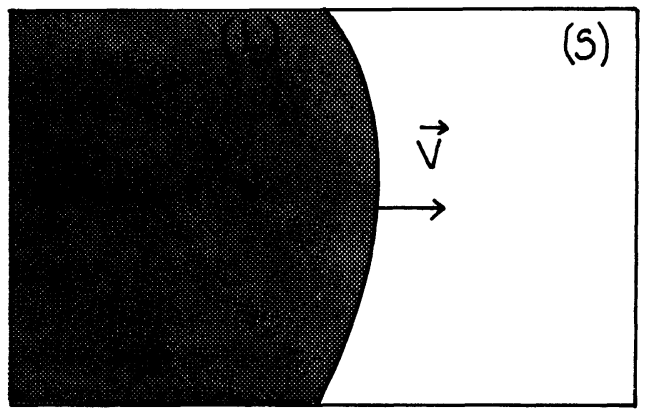

Fig. 8. - Méthode simplifiée : principe de calcul.

[Simplified method : the calculation principle.] 
En faisant varier $P_{0}$, on en déduit finalement toutes les positions de l'interface pour l'ensemble des valeurs prises par le couple $(\Phi, V)$. $\Phi$ et $V$ sont alors des paramètres parfaitement substituables. Cette méthode n'est facilement applicable que si la forme de l'interface dépend essentiellement de sa position, et qu'on peut négliger le changement de forme dû à $V$.

4. Application à un problème unidimensionnel. 4.1 CONVERGENCE Vers LA SOlUtion ANALYTIQUE. La méthode précédente a été testée sur un problème unidimensionnel pour lequel existe une solution analytique $[6,7]:$ le cas du lingot tiré à partir d'un bain fondu.

Si ce lingot est un ruban d'épaisseur constante $e$, négligeable devant sa largeur, si sa longueur est infinie (dans la pratique supérieure à quelques centimètres), si de plus la vitesse de tirage est nulle, le gradient dans le solide à l'interface a l'expression simple suivante :

$$
G_{\mathrm{s}}=\left|\left(\frac{\partial u}{\partial n}\right)_{\mathrm{s}}\right|=\frac{2}{\sqrt{e}} \sqrt{\frac{\varepsilon \sigma b u_{\mathrm{F}}}{\lambda_{\mathrm{F}}}}
$$

$\lambda_{\mathrm{F}}$ : conductivité du solide au point de fusion.

$$
b=\frac{u_{\mathrm{F}}^{4}-u_{0}^{4}}{4}-u_{0}^{4} \operatorname{Ln}\left(\frac{u_{\mathrm{F}}}{u_{0}}\right) .
$$

Dans le cas du silicium :

$$
\begin{aligned}
\varepsilon & =0,5 \\
u_{\mathrm{F}} & =1683 \mathrm{~K} \\
\lambda_{\mathrm{F}} & =0,05 \mathrm{cal} . \mathrm{cm}^{-1} \mathrm{~s}^{-1} \mathrm{~K}^{-1} .
\end{aligned}
$$

De sorte que : $G_{\mathrm{s}}=\frac{132}{\sqrt{e}} \mathrm{~K} \mathrm{~mm}^{-1}(e$ en $\mathrm{mm})$.

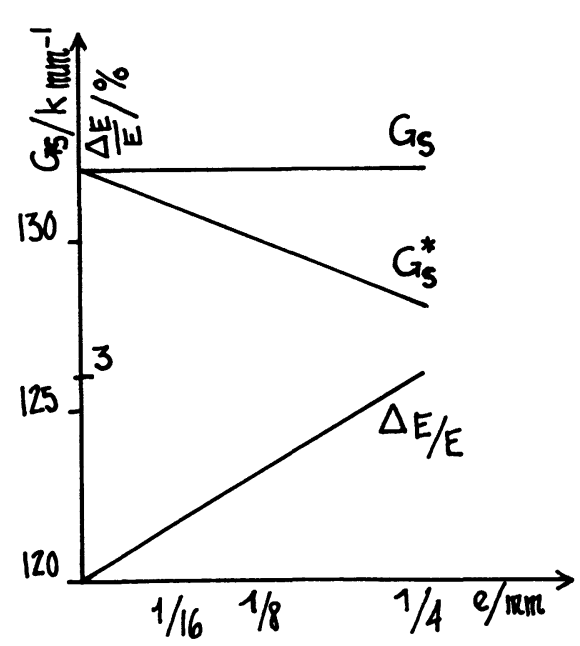

Fig. 9. - Convergence de $G_{\mathrm{s}}^{*}$ numérique vers $G_{\mathrm{s}}$ analytique. Diminution de l'erreur relative sur la conservation de la puissance $\Delta E / E$.

[Convergence of numerical $G_{\mathrm{s}}^{*}$ to analytical $G_{\mathrm{s}}$. Decreasing of the relative error on the power conservation $(\Delta E / E)$.]
Le modèle précédent a été utilisé avec un pas de maillage de $1 / 4 \mathrm{~mm}, 1 / 8 \mathrm{~mm}, 1 / 16 \mathrm{~mm}$. On a pu vérifier sa convergence vers la solution analytique (Fig. 9). L'erreur relative sur la conservation de la puissance $\Delta E / E$ tend vers zéro, le modèle étant d'autant plus précis que le maillage est fin.

\subsection{Avantages de La MÉTHOde PROPOSÉE. - Dans} le cas particulier du tirage de ruban à une dimension, la méthode numérique permet d'effectuer de nombreux calculs qui n'admettent pas de solution analytique :

- calcul explicite des températures dans le solide ou le liquide,

- influence des paramètres du liquide sur la hauteur de zone flottante,

- influence de la longueur du ruban,

- influence d'une variation locale des dimensions du ruban (épaisseur et largeur).

Ce dernier point est le plus intéressant puisque, combiné avec une étude hydrostatique, il rend possible une étude du comportement quasi stationnaire ou transitoire du tirage d'un ruban.

5. Application au cas du cylindre en rotation. 5.1 Mise en ÉQuation. - L'équation (3) a ici la forme suivante :

$$
-\lambda_{\mathrm{s}}\left(\frac{\partial u}{\partial n}\right)_{\mathrm{s}}=-\lambda_{1}\left(\frac{\partial u}{\partial n}\right)_{1}+\rho_{\mathrm{s}} \operatorname{L\omega r(\mathbf {n.v})}
$$

n : vecteur unitaire normal à l'interface au point considéré,

$\mathbf{v}$ : vecteur unitaire orthoradial en ce même point.

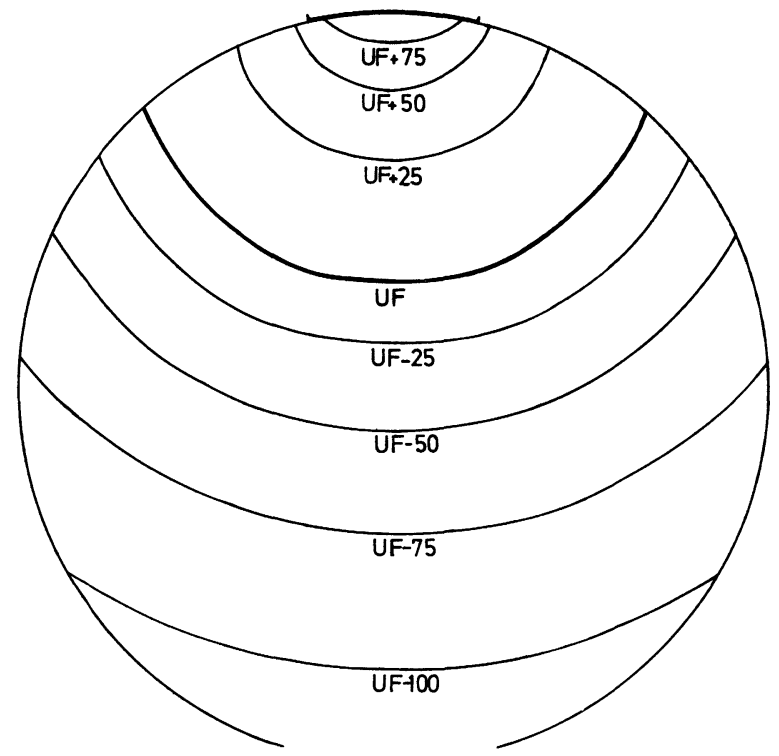

Fig. 10. - Forme des isothermes pour $\Phi=240 \mathrm{~W}$ et $\omega=0$. [Isotherms shapes for $\Phi=240 \mathrm{~W}$ and $\omega=0$.] 




Fig. 11. - Forme des isothermes pour $\Phi=240 \mathrm{~W}$ et $\omega=0,15 \mathrm{t} / \mathrm{min}$.

[Isotherms shapes for $\Phi=240 \mathrm{~W}$ and $\omega=0.15 \mathrm{t} / \mathrm{min}$.]

Les équations (1), (2), (4) ainsi que les conditions aux limites à la circonférence du cylindre ont été résolues par la méthode exposée en 3.2.

Le critère de convergence est la conservation de la puissance. Explicitons-le :

Puissance incidente :

$$
\Phi=\Phi_{\mathrm{s}} \delta l .
$$

Puissance rayonnée :

$$
\Phi^{\prime}=\sum_{\mathrm{i}, \mathrm{s}} \varepsilon_{\mathrm{s}} \sigma\left(u_{\mathrm{i}}^{4}-u_{0}^{4}\right) \mathrm{d} s+\sum_{\mathrm{i}, 1} \varepsilon_{1} \sigma\left(u_{\mathrm{i}}^{4}-u_{0}^{4}\right) \mathrm{d} s
$$

$\sum_{\mathrm{i}, \mathrm{s}}\left(\right.$ respectivement $\left.\sum_{\mathrm{i}, \mathrm{i}}\right)$ représentant une sommation sur toutes les mailles de la partie solide (respectivement liquide) de la circonférence.

Le calcul est considéré còmme convergent dès que :

$$
\frac{2\left|\Phi-\Phi^{\prime}\right|}{\Phi+\Phi^{\prime}}<P \quad(P \text { précision fixée a priori }) .
$$

5.2 Résultats. - Sur la figure 10, ont été tracés les isothermes (tous les $25 \mathrm{~K}$ ) dans la partie solide et liquide pour $\Phi=240 \mathrm{~W}$ et $\omega=0$. Et sur la figure 11, pour $\Phi=240 \mathrm{~W}$ et $\omega=0,15 \mathrm{t} / \mathrm{min}$.

5.3 INTERPRÉTATION. - Sur le cylindre au repos, on observe une discontinuité du gradient à l'interface due au changement de conductivité.

On observe, même à faible vitesse de rotation, une déformation importante des isothermes. Cette déformation est due à la chaleur latente de fusion du silicium qui est très élevée (de l'ordre de $430 \mathrm{cal}^{\mathrm{g}} \mathrm{g}^{-1}$ ). Comme on le voit sur la figure 12, la déformation

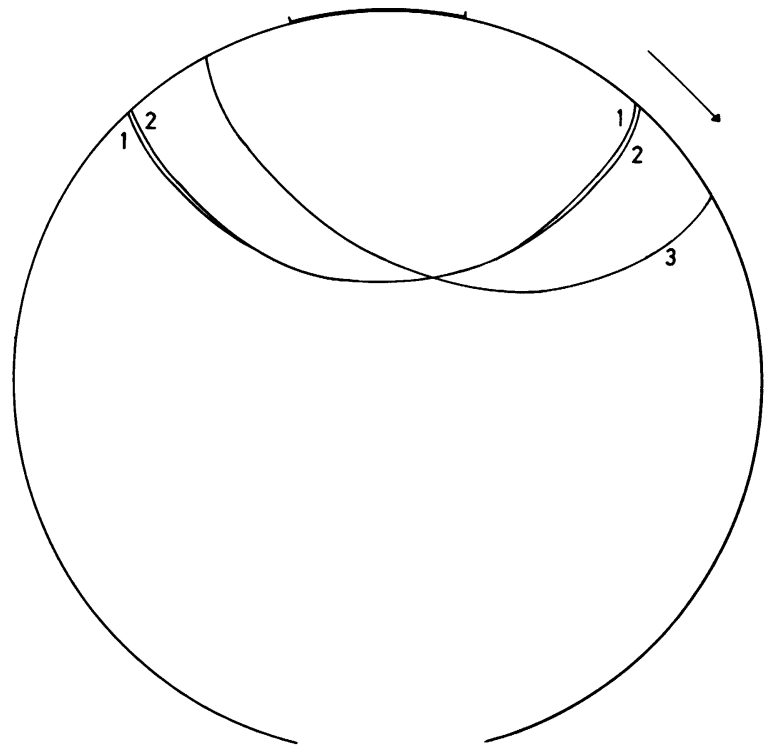

Fig. 12. - Comparaison de la forme de l'interface pour : $1 \omega=0.2 \omega=0,15 \mathrm{t} / \mathrm{min}$. $L=0.3 \omega=0,15 \mathrm{t} / \mathrm{min}$; $L=430$ cal. $\mathrm{g}^{-1}$.

[Comparison of the isotherms shape for : $1 \omega=0$. 2 $\omega=0.15 \mathrm{t} / \mathrm{min}$. $L=0.3 \omega=0.15 \mathrm{t} / \mathrm{min}$. $L=430 \mathrm{cal}$. $\mathrm{g}^{-1}$.]

supplémentaire due à la chaleur massique est très faible à cette même vitesse de rotation.

Le déplacement de l'interface de cristallisation (qui s'effectue dans le sens des gradients décroissants) est plus important que celui de l'interface de fusion (sens des gradients croissants).

Le gradient orthoradial à la surface du liquide à l'interface de cristallisation diminue avec la vitesse de rotation, tandis que celui de l'interface de fusion augmente avec la vitesse de rotation. Pour une certaine valeur de $\omega$, la libération de chaleur latente à l'interface de cristallisation est telle qu'elle annule le gradient dans le liquide. Pour $\omega$ plus élevé, il y a alors surfusion, et cristallisation très rapide du liquide sous forme de dendrites. La figure 11 correspond à cette valeur limite de $\omega$.

Cette vitesse limite existe dans tout processus de croissance de cristaux à partir d'un bain fondu, et avait déjà été mise en évidence dans le cas d'un tirage de lingot [6,7]. Dans le cas du cylindre, on peut évaluer $\omega_{\text {lim }}$ par la formule :

$$
\omega_{\lim }=\frac{\lambda_{\mathrm{s}} G_{\mathrm{s}}}{\rho_{\mathrm{s}} L R(\mathbf{n . v})}
$$

où

$$
G_{\mathrm{s}}=\left|\left(\frac{\partial u}{\partial n}\right)_{\mathrm{s}}\right|
$$

$\omega_{\text {lim }}$ dépend de $R$ et de $\Phi$ par l'intermédiaire de $G_{s}$. $\mathrm{Du}$ fait de la chaleur latente de fusion très élevée du silicium, le débit thermique d'alimentation de la 
zone fondue est important. Evaluons-le dans le cas de la figure 11 :

$$
D=\rho_{\mathrm{s}} L l \int_{\Sigma} \mathbf{v}_{\mathbf{M}} \mathbf{n} \mathrm{d} s
$$

$\mathbf{n}$ : normale à l'interface en $\mathbf{M} ; \mathbf{s}:$ abscisse curviligne ;

$\Sigma:$ demi-interface

$D=\rho_{\mathrm{s}} L l \int_{R-\Delta R}^{R} \omega r \mathrm{~d} r=\rho_{\mathrm{s}} L l \omega \Delta R(R-\Delta R / 2)$

$R$ : rayon du cylindre;

$\Delta R$ : profondeur de la zone fondue.

On trouve $D=50 \mathrm{~W}$, soit près de $20 \%$ de la puissance incidente.

- Bilan thermique global correspondant à la figure 11 :

Puissance incidente : $\Phi=240 \mathrm{~W}$.

Puissance rayonnée par la zone fondue : $\Phi_{1}=50 \mathrm{~W}$.

Puissance rayonnée par la partie solide : $\Phi_{2}=190 \mathrm{~W}$.

Puissance absorbée-libérée aux interfaces :

$$
D= \pm 50 \mathrm{~W} \text {. }
$$

- Conservation de la puissance : $\Phi=\Phi_{1}+\Phi_{2}$.

Conservation de la quantité de matière fondue : débit massique de fusion $=$ débit massique de cristallisation.

6. Conclusions. - On a exposé une méthode de calcul numérique très générale d'un système thermique en mouvement avec changement d'état.

Cette méthode a permis d'étudier l'équilibre d'une zone fondue sur un cylindre en rotation. On a ainsi par exemple mis en évidence une vitesse limite de rotation au-delà de laquelle le gradient à la surface du liquide à l'interface de cristallisation change de signe. Cette vitesse limite dépend du rayon du cylindre et de la puissance de chauffage.

Ce modèle, que l'on devra comparer avec nos résultats expérimentaux, est d'ores et déjà très utile pour comprendre le comportement thermique d'un tel système et interpréter les premiers essais de cristallisation de rubans effectués à partir d'une telle zone fondue.

\section{Bibliographie}

[1] Rodot, H., Cassagne, P., Hamidi, M., Theoretical studies on a new single crystalline ribbon growth method. J. Electron. Mater. 10 no 3 (1981) 481.

[2] Carslaw, M. S., Jaeger, J. C., Conduction of heat in solids (University Press, Oxford) 1959.

[3] Price, P. H., Slack, M. R., Brit. J. Appl. Phys. 5 (1954) 285.

[4] Crank, J., Quart. J. Mech. Appl. Math. 10 (1957) 220.

[5] Bell, R. O., Muller, J. C., Toulemonde, M., Stich, R.,
SIFFerT, P., Effects of ruby laser pulses on virgin and amorphous silicon surface layers. Laser solid interactions and laser processing 28, 11-1 12.1978 (Boston).

[6] Zook, J. D., Schuldt, S. B., Analysis of conditions for high speed growth of silicon sheet. Crystal Growth 50 (1980) 51.

[7] RAVI, K. V., The growth of EFG silicon ribbons. Crystal Growth 39 (1977) 1. 